\title{
Gaussian Process Model Based Predictive Control
}

\author{
Juš Kocijan, Roderick Murray-Smith, \\ Carl Edward Rasmussen, Agathe Girard
}

\begin{abstract}
Gaussian process models provide a probabilistic non-parametric modelling approach for black-box identification of non-linear dynamic systems. The Gaussian processes can highlight areas of the input space where prediction quality is poor, due to the lack of data or its complexity, by indicating the higher variance around the predicted mean. Gaussian process models contain noticeably less coefficients to be optimized. This paper illustrates possible application of Gaussian process models within model-based predictive control. The extra information provided within Gaussian process model is used in predictive control, where optimization of control signal takes the variance information into account. The predictive control principle is demonstrated on control of $\mathrm{pH}$ process benchmark.
\end{abstract}

\section{INTRODUCTION}

Model Predictive Control (MPC) is a common name for computer control algorithms that use an explicit process model to predict the future plant response. According to this prediction in the chosen period, also known as the prediction horizon, the MPC algorithm optimizes the manipulated variable to obtain an optimal future plant response. The input of chosen length, also known as control horizon, is sent into the plant and then the entire sequence is repeated again in the next time period. The popularity of MPC is to a great extent owed to the ability of MPC algorithms to deal with constraints that are frequently met in control practice and are often not well addressed with other approaches. MPC algorithms can handle hard state and rate constraints on inputs and states that are usually, but not always incorporated in the algorithms via an optimization method. Linear model based predictive control approaches [12] started appearing in the early eighties and are well-established in control practice (e.g. overview in [17]). Nonlinear model based predictive control (NMPC) approaches [1] start to appear about ten years later and have also found their way into control practice (e.g. [18], [22]) though its popularity can not be compared to linear model based predictive control. This fact is very likely connected with difficulty in nonlinear model construction and with lack of necessary trust in this model. There were a number of contributions in the field of nonlinear model based predictive control dealing with

J. Kocijan is with Jozef Stefan Institute, Jamova 39, SI-1000 Ljubljana, Slovenia and Nova Gorica Polytechnic, Nova Gorica, Slovenia jus.kocijaneijs.si

R. Murray-Smith is with the Department of Computing Science, University of Glasgow, Glasgow, United Kingdom and Hamilton Institute, National University of Ireland, Maynooth, Ireland rodedcs.gla.ac.uk

C. E. Rasmussen is with ax Planck Institute for Biological Cybernetics, Tübingen, Germany carl@tuebingen.mpg.de

A. Girard is with the Department of Computing Science, University of Glasgow, Glasgow, United Kingdom agatheedcs.gla.ac.uk issues like stability, efficient computation, optimization, constraints and others. Some recent work in this field can be found in [2], [11]. NMPC algorithms are based on various nonlinear models. Often these models are developed as first principles models, but other approaches, like black-box identification approaches are also popular. Various predictive control algorithms are based on neural networks model e.g. [16], fuzzy models e.g. [7] or local model networks e.g. [5]. The quality of control depends on quality of model. New developments in NMPC approaches are coming from resolving various issues: from faster optimization methods to different process model. The contribution of this paper is to describe a NMPC principle with a Gaussian process model. The Gaussian process model is an example of a probabilistic non-parametric model that also provides information about prediction uncertainties which are difficult to evaluate appropriately in nonlinear parametric models. The majority of work on Gaussian processes shown up to now considers modelling of static non-linearities. The use of Gaussian processes in modelling dynamic systems is a recent development e.g. [14], [13], [3], [20], [9], [10] and some control algorithms based on such are described in [15], [4].

The paper is organized as follows. Dynamic Gaussian process models are described in the next section. The control algorithm principle is described in Section III and illustrated with the benchmark $\mathrm{pH}$ process control in Section IV. Conclusions are stated at the end of the paper.

\section{MODELLING OF DYNAMIC SYSTEMS WITH GAUSSIAN PROCESSES}

A Gaussian process is an example of the use of a flexible, probabilistic, non-parametric model with uncertainty predictions. Its use and properties for modelling are reviewed in [21].

A Gaussian process is a collection of random variables which have a joint multivariate Gaussian distribution. Assuming a relationship of the form $y=f(\mathbf{x})$ between an input $\mathbf{x}$ and output $y$, we have $y^{1}, \ldots, y^{n} \sim \mathcal{N}(0, \Sigma)$, where $\Sigma_{p q}=\operatorname{Cov}\left(y_{p}, y_{q}\right)=C\left(\mathbf{x}_{p}, \mathbf{x}_{q}\right)$ gives the covariance between output points corresponding to input points $\mathbf{x}_{p}$ and $\mathbf{x}_{q}$. Thus, the mean $\mu(\mathbf{x})$ (usually assumed to be zero) and the covariance function $C\left(\mathbf{x}_{p}, \mathbf{x}_{q}\right)$ fully specify the Gaussian process. Note that the covariance function $C(.,$. can be any function with the property that it generates a positive definite covariance matrix. 
A common choice is

$$
C\left(\mathbf{x}_{p}, \mathbf{x}_{q}\right)=v_{1} \exp \left[-\frac{1}{2} \sum_{d=1}^{D} w_{d}\left(x_{p}^{d}-x_{q}^{d}\right)^{2}\right]+v_{0}
$$

where $\boldsymbol{\Theta}=\left[\begin{array}{llll}w_{1} \ldots w_{D} & v_{0} & v_{1}\end{array}\right]^{T}$ are the 'hyperparameters' of the covariance functions and $D$ the input dimension. Other forms of covariance functions suitable for different applications can be found in [19]. For a given problem, the parameters are learned (identified) using the data at hand. After the learning, one can use the $w$ parameters as indicators of 'how important' the corresponding input components (dimensions) are: if $w_{d}$ is zero or near zero it means that the inputs in dimension $d$ contain little information and could possibly be removed.

Consider a set of $N D$-dimensional input vectors $\mathbf{X}=\left[\mathbf{x}_{1}, \mathbf{x}_{2}, \ldots, \mathbf{x}_{N}\right]$ and a vector of output data $\mathbf{y}=$ $\left[y^{1}, y^{2}, \ldots, y^{N}\right]^{T}$. Based on the data $(\mathbf{X}, \mathbf{y})$, and given a new input vector $\mathrm{x}^{*}$, we wish to find the predictive distribution of the corresponding output $y^{*}$. Unlike other models, there is no model parameter determination as such, within a fixed model structure. With this model, most of the effort consists in tuning the parameters of the covariance function. This is done by maximizing the log-likelihood of the parameters, which is computationally relatively demanding since the inverse of the data covariance matrix $(N \times N)$ has to be calculated at every iteration.

The described approach can be easily utilized for regression calculation. Based on training set $\mathbf{X}$ a covariance matrix $\mathbf{K}$ of size $N \times N$ is determined. As already mentioned before the aim is to find the distribution of the corresponding output $y^{*}$ at some new input vector $\mathbf{x}^{*}=\left[x_{1}(N+1), x_{2}(N+1), \ldots, x_{D}(N+1)\right]^{T}$.

For a new test input $\mathrm{x}^{*}$, the predictive distribution of the corresponding output is $y^{*} \mid \mathbf{x}^{*},(\mathbf{X}, \mathbf{y})$ and is Gaussian, with mean and variance

$$
\begin{aligned}
\mu\left(\mathbf{x}^{*}\right) & =\mathbf{k}\left(\mathbf{x}^{*}\right)^{T} K^{-1} \mathbf{y} \\
\sigma^{2}\left(\mathbf{x}^{*}\right) & =k\left(\mathbf{x}^{*}\right)-\mathbf{k}\left(\mathbf{x}^{*}\right)^{T} \mathbf{K}^{-1} \mathbf{k}\left(\mathbf{x}^{*}\right)+v_{0}
\end{aligned}
$$

where $\mathbf{k}\left(\mathbf{x}^{*}\right)=\left[C\left(\mathbf{x}^{1}, \mathbf{x}^{*}\right), \ldots, C\left(\mathbf{x}^{N}, \mathbf{x}^{*}\right)\right]^{T}$ is the $N \times 1$ vector of covariances between the test and training cases and $k\left(\mathbf{x}^{*}\right)=C\left(\mathbf{x}^{*}, \mathbf{x}^{*}\right)$ is the covariance between the test input and itself.

For multi-step ahead prediction we have to take account of the uncertainty of future predictions which provide the 'inputs' for estimating further means and uncertainties.

If we now consider a new random input, $\mathbf{x}^{*} \sim$ $\mathcal{N}\left(\mu_{x^{*}}, \Sigma_{x^{*}}\right)$, Girard et. al. [3], have shown that, within a Gaussian approximation and a Taylor expansion $\mu\left(\mathbf{x}^{*}\right)$ and $\sigma^{2}\left(\mathbf{x}^{*}\right)$ around $\mu_{x^{*}}$, the predictive distribution is again Gaussian with mean and variance

$$
\begin{aligned}
m\left(\mathbf{x}^{*}\right) & =E_{\mathbf{x}^{*}}\left[\mu\left(\mathbf{x}^{*}\right)\right] \\
& \approx \mathbf{k}\left(\mu\left(\mathbf{x}^{*}\right)^{T} \mathbf{K}^{-1} \mathbf{y}\right. \\
v\left(\mathbf{x}^{*}\right) & =E_{\mathbf{x}^{*}}\left[\sigma^{2}\left(\mathbf{x}^{*}\right)\right]+\operatorname{var}_{\mathbf{x}^{*}}\left(\mu\left(\mathbf{x}^{*}\right)\right) \\
& \approx \sigma^{2}\left(\mu\left(\mathbf{x}^{*}\right)\right) \\
& +\operatorname{trace}\left\{\Sigma _ { \mathbf { x } ^ { * } } \left(\left.\frac{1}{2} \frac{\partial^{2} \sigma^{2}\left(\mathbf{x}^{*}\right)}{\partial \mathbf{x}^{*} \partial \mathbf{x}^{* T}}\right|_{\mathbf{x}^{*}=\mu\left(\mathbf{x}^{*}\right)}\right.\right. \\
& \left.\left.+\left.\left.\frac{\partial \mu\left(\mathbf{x}^{*}\right)}{\partial \mathbf{x}^{*}}\right|_{\mathbf{x}^{*}=\mu\left(\mathbf{x}^{*}\right)} \frac{\partial \mu\left(\mathbf{x}^{*}\right)}{\partial \mathbf{x}^{*}}\right|_{\mathbf{x}^{*}=\mu\left(\mathbf{x}^{*}\right)} ^{T}\right)\right\}
\end{aligned}
$$

For a more detailed derivation see [3]. Equations (4) and (5) can be applied to calculation of multi-step ahead prediction with propagation of uncertainty.

Gaussian processes can, like neural networks, be used to model static nonlinearities and can therefore be used for modelling of dynamic systems if delayed input and output signals are fed back and used as regressors. In such cases an autoregressive model is considered, such that the current output depends on previous outputs, as well as on previous control inputs.

$$
\begin{aligned}
\mathbf{x}(k)= & {[\hat{y}(k-1), \hat{y}(k-2), \ldots, \hat{y}(k-L), u(k-1),} \\
& u(k-2), \ldots, u(k-L)]^{T} \\
\hat{y}(k)= & f(\mathbf{x}(k))+\epsilon
\end{aligned}
$$

Where $k$ denotes consecutive number of data sample. Let $\mathbf{x}$ denote the state vector composed of the previous outputs $y$ and inputs $u$ up to a given lag $L$ and $\epsilon$ is white noise.

It is worthwhile noting that the derivatives of means and variances can be calculated in straightforward manner. For more details see [20] or [3].

As can be seen from the presented relations the obtained model describes both the dynamic characteristics of nonlinear system, and at the same time provides information about the confidence in these predictions. The Gaussian process can highlight areas of the input space where prediction quality is poor, due to the lack of data, by indicating the higher variance around the predicted mean.

\section{NONLINEAR MODEL PREDICTIVE CONTROL}

Nonlinear model predictive control as it was applied with the Gaussian process model can be in general described with a block diagram, as depicted in Figure 1. The model used is fixed, identified off-line, which means that used control algorithm is not an adaptive one. The structure of the entire control loop is therefore less complex as in the case where used model changes with time. The following items describe the basic idea of predictive control:

- Prediction of system output signal $y(k+j)$ is calculated for each discrete sample $k$ for a large horizon in future $\left(j=N_{1}, \ldots, N_{2}\right)$. Predictions are denoted as $\hat{y}(k+j \mid k)$ and represent $j$-step ahead prediction, 


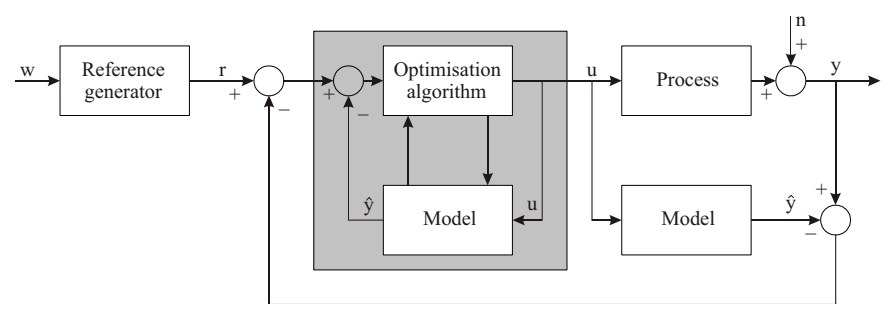

Fig. 1. Block diagram of model predictive control system

while $N_{1}$ and $N_{2}$ determine lower and upper bound of prediction horizon. Lower and upper bound of output signal prediction horizon determine coincidence horizon, within which a match between output and reference signal is expected. Output signal prediction is calculated from process model. Predictions are dependent also on the control scenario in the future $u(k+j \mid k), j=0, \ldots, N_{u}-1$, which is intended to be applied from a moment $k$ onwards.

- The reference trajectory is determined $r(k+j \mid k), j=$ $N_{1}, \ldots, N_{2}$, which determines reference process response from present value $y(k)$ to the setpoint trajectory $w(k)$.

- The vector of future control signals $(u(k+j \mid k), j=$ $\left.0, \ldots, N_{u}-1\right)$ is calculated by minimization of objective function such that predicted error between $r(k+j \mid k)$ and $\hat{y}(k+j \mid k), j=N_{1}, \ldots, N_{2}$ is minimal. Structuring of future control samples can be used in some approaches.

- Only the first element $u(k \mid k)$ of the optimal control signal vector $u(k+j \mid k), j=0, \ldots, N_{u}-1$ is applied.

In the next sample a new measured output sample is available and the entire described procedure is repeated. This principle is called receding horizon strategy.

A moving-horizon minimization problem of the special form [12]

$$
\min _{\mathbf{U}(k)}[r(k+P)-\hat{y}(k+P)]^{2}
$$

subject to:

$$
\begin{aligned}
\operatorname{var} \hat{y}(k+P) & \leq k_{v} \\
|\mathbf{U}(k)| & \leq k_{i h} \\
|\dot{\mathbf{U}}(k)| & \leq k_{i r} \\
|\mathbf{x}(k)| & \leq k_{s h} \\
|\dot{\mathbf{x}}(k)| & \leq k_{s r}
\end{aligned}
$$

is used in our case, where $\mathbf{U}(k)=[u(k) \ldots u(k+P)]$ is input signal, $P$ is the coincidence point (the point where a match between output and reference value is expected) and inequalities from (8) to (12) represent constraint on output variance $k_{v}$, input hard constraint $k_{i h}$, input rate constraint $k_{i r}$, state hard constraint $k_{s h}$ and state rate constraint $k_{s r}$ respectively. The process model is a Gaussian process.

The optimization algorithm, which is constrained nonlinear programming, is solved at each sample time over a prediction horizon of length $P$, for a series of moves which equals to control horizon. In our case control horizon was chosen to be one and to demonstrate constraint on variance the rest of constraints was not taken into the account. Nevertheless, all this modifications do not change the generality of solution, but they do affect the numerical solution itself.

Alternative ways of how NMPC with Gaussian process models can be realized are as follows.

- Different objective function: The objective function used (7) is just one of many possible ones. It is well known that selection of the objective function has a major impact on the amount of computation.

- Optimization problem for $\Delta \mathbf{U}(k)$ instead of $\mathbf{U}(k)]$ : This is not just a change of formalism, but also enables other forms of MPC. One possibility is a DMC controller with nonlinear model, e.g. [7] - a frequently used principle, that together with appropriate objective function enables problem representation as a least squares problem that can be solved in one iteration in which an explicit solution is found. This is, as in the case with other special case simplifications, not a general case solution.

- Soft constraints: Using constraint optimization algorithms is very demanding for computation and soft constrains, namely weights on constrained variables in objective function, can be used to decrease the amount of computation. More on this topic can be found in [8], [23].

- Linear MPC: It is worth to remark that even though this is a constrained nonlinear MPC problem it can be used in its specialized form as a robust linear MPC.

There are several issues of interest for applied NMPC. Let us mention some of them. One of them is efficient numerical solution. Nonlinear programming optimization algorithm is very demanding for computation. Various approximations and other approaches (e.g. approximation of explicit solution) exist to decrease computational load, mainly for special cases, like linear process models or special objective functions.

One possibility to decrease the computational load necessary for optimization is with the incorporation of prediction derivation (and variance) into optimization algorithm. When using Gaussian process models the prediction and variance derivation can be calculated in a straightforward manner.

Stability of the closed-loop systems is next issue. At present no stability conditions have been derived for Gaussian processes as a representative of probabilistic nonparametric models.

Applied control can not avoid issue of control system robustness. This issue has a major impact on the applicability of the algorithm in practice. The fact that the process model contains the information about the model confidence enables controller to optimize the manipulative variable to "avoid" regions where the confidence in model is not high enough. This possibility itself makes the controller robust 
if applied properly. MPC robustness in the case of other algorithms is usually not some specially built feature of the MPC algorithms, but was more an issue of assessment for particular MPC algorithms.

\section{EXAMPLE}

\section{A. $p H$ process}

A simplified schematic diagram of the $\mathrm{pH}$ neutralization process taken from [6] is given in Figure 2. The process consists of an acid stream $\left(Q_{1}\right)$, buffer stream $\left(Q_{2}\right)$ and base stream $\left(Q_{3}\right)$ that are mixed in a tank $\mathrm{T}_{1}$. Prior to mixing, the acid stream enters the tank $\mathrm{T}_{2}$ which introduces additional flow dynamics. The acid and base flow rates are controlled with flow control valves, while the buffer flow rate is controlled manually with a rotameter. The effluent $\mathrm{pH}(p H)$ is the measured variable. Since the $\mathrm{pH}$ probe is located downstream from the tank $\mathrm{T}_{1}$, a time delay $\left(T_{d}\right)$ is introduced in the $\mathrm{pH}$ measurement. In this study, the $\mathrm{pH}$ is controlled by manipulating the base flow rate. A more detailed description of the process with mathematical model and necessary parameters is presented in [6].

The dynamic model of the $\mathrm{pH}$ neutralization system shown in Fig. 2 is derived using the conservation equations and equilibrium relations. The model also includes valve and transmitter dynamics as well as hydraulic relationships for the tank outlet flows. Modelling assumptions include perfect mixing, constant density, and complete solubility of the ions involved. The simulation model of $\mathrm{pH}$ process, which was used for necessary data generation contains therefore various non-linear elements as well as implicitly calculated function which is value of highly non-linear titration curve.

\section{B. Model identification}

Based on responses and iterative cut-and-try procedure a sampling time of 25 seconds was selected. The sampling

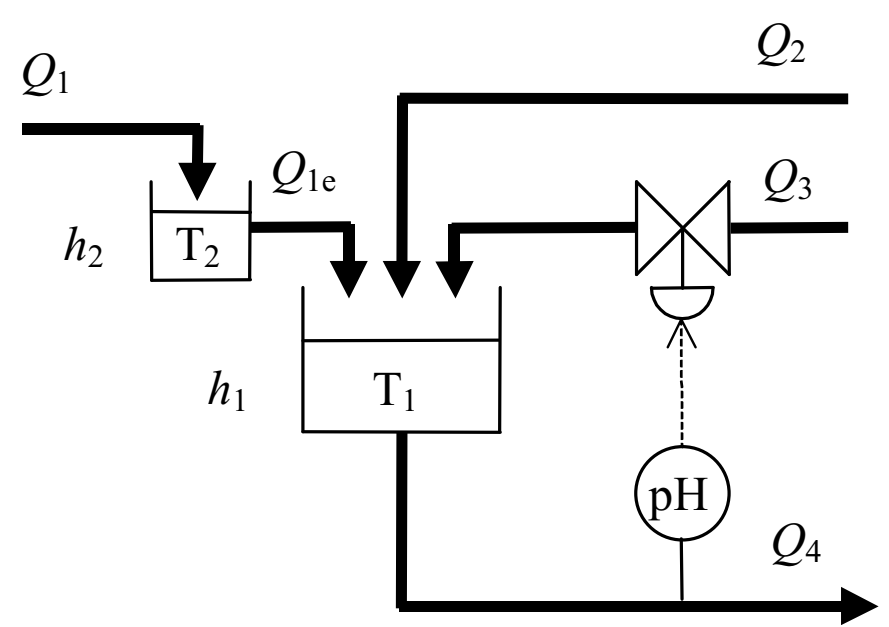

Fig. 2. The pH neutralization system scheme time was so large that the dead-time mentioned in the previous section disappeared.

The chosen identification signal of 400 samples was generated from a uniform random distribution and rate of 50 seconds.

Obtained hyperparameters of the third order Gaussian process model were:

$$
\begin{aligned}
\boldsymbol{\Theta}= & {\left[w_{1}, w_{2}, w_{3}, w_{4}, w_{5}, w_{6}, v_{0}, v_{1}\right] } \\
= & {[-6.0505,-2.0823,-0.4785,-5.3388,-3.4206,} \\
& -8.7080,0.8754,-5.4164]
\end{aligned}
$$

where hyperparameters from $w_{1}$ to $w_{3}$ denote a weight for each output regressor, from $w_{4}$ to $w_{6}$ denote a weight for each input regressor, $v_{0}$ is estimated noise variance and $v_{1}$ is the estimate of the vertical variance.

The region in which the model was obtained can be seen from Figure 3. A very good fit can be observed for the identification input signal which was used for optimization. However, the obtained model contains information mainly in the region below $\mathrm{pH}=7$ as can be concluded from the response in Figure 3. The validation signal had lower magnitude and frequency components than the identification signal. The rationale behind this is that if the identified model was excited with a richer signal, than it has to respond well to the signal with less components. The validation signal was obtained with generator of random noise with uniform distribution and rate of 500 seconds. Response of the model to validation signal and comparison with process response is depicted in Figure 4. Fitting of the response for validation signal:

- average absolute test error

$$
A E=0.1276
$$

- average squared test error

$$
S E=0.0373
$$

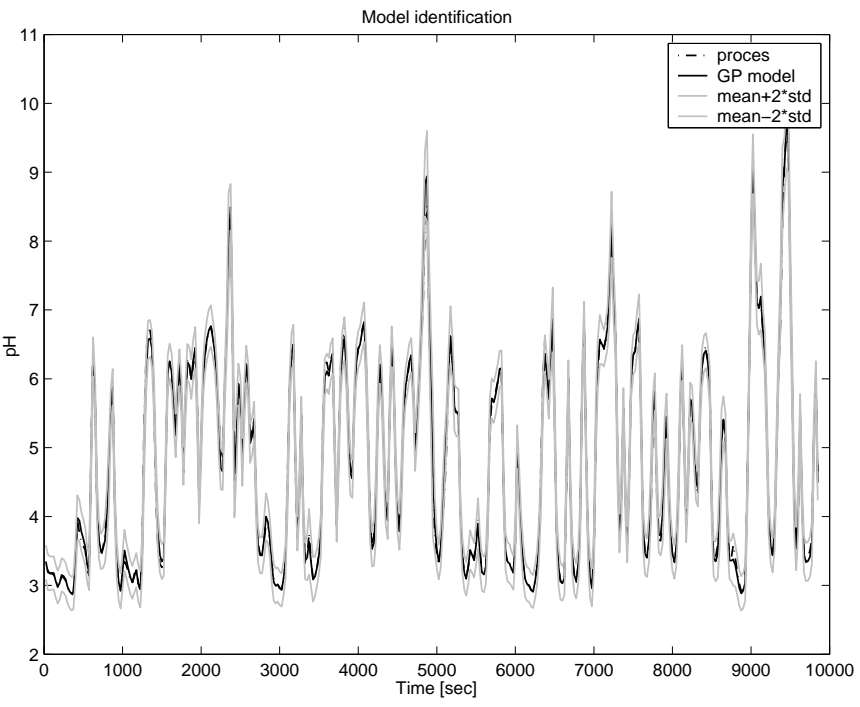

Fig. 3. Response of GP model on excitation signal used for identification 


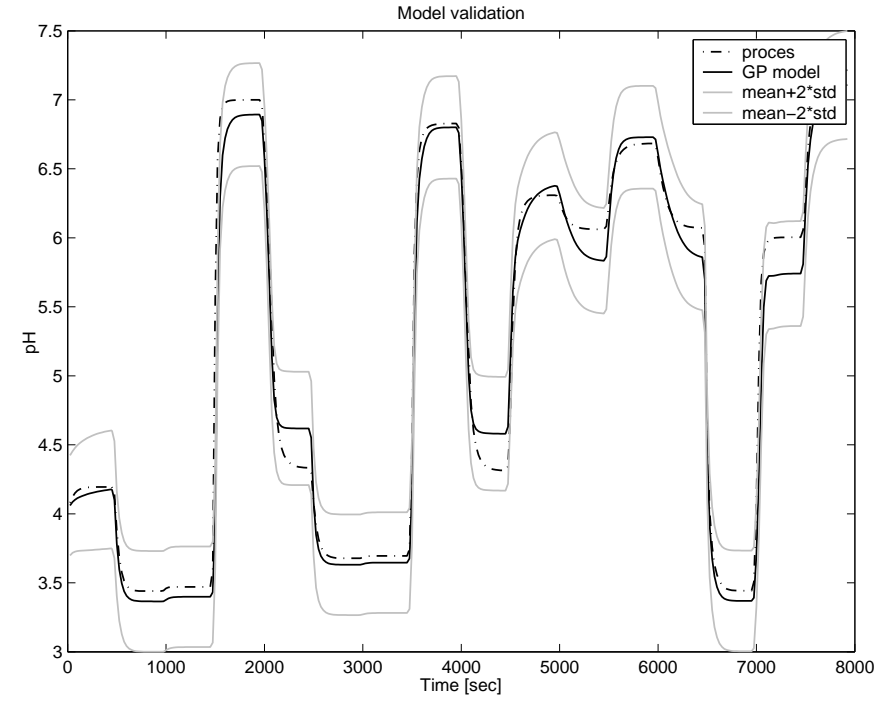

Fig. 4. Response of GP model on excitation signal used for validation

- $\log$ density error

$$
L D=1.9889
$$

After model validation the model was utilized for control design. See [10] for more issues on $\mathrm{pH}$ process modelling.

\section{Control}

The control algorithm described above was tested for the $\mathrm{pH}$ process with simulation. The reference trajectory $r$ is defined so that it approaches the set-point exponentially from the current output value. The coincidence point was chosen to be 8 samples and, as already mentioned, the control horizon is one sample. The results of unconstrained control are given in Figures 5 and 6.
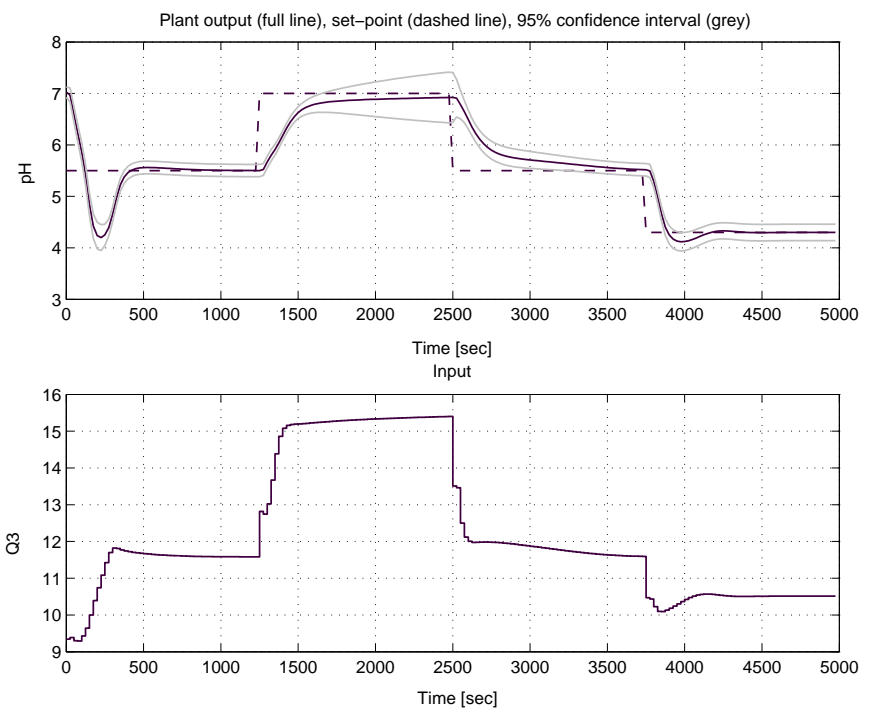

Fig. 5. Non-constrained case: response of GP model based control (upper figure) and control signal (bottom figure)

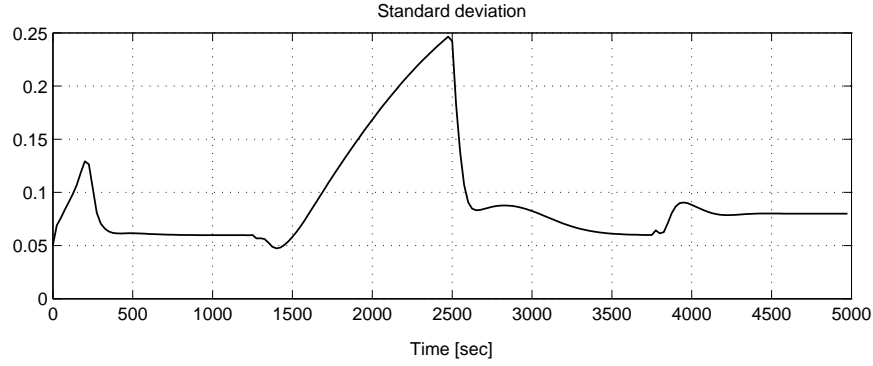

Fig. 6. Non-constrained case: standard deviation corresponding to the previous figure

It can be seen from different set-point responses that the model differs from the process in different regions. It can be clearly seen that the variance increases as output signal approaches regions which were not populated with enough identification data. It should be noted however that variances are sum of variances that correspond to information about regions where model is more or less to be trusted depending upon available identification data and of output response variances that indicate model quality. When variances increase too much, the response can be optimized with constrained control. Results can be seen in Figures 7 and 8 .

It can be seen from Figs. 7 and 8 that the closed-loop system response avoids region with large variance at the cost of steady-state error. This could be interpreted also as trade-off between designed performance and safety.

\section{CONCLUSIONS}

The principle of Model Predictive Control based on a Gaussian process model was presented in the paper and illustrated with $\mathrm{pH}$ process control example. In the example, a constraint on model variance was included. This can be
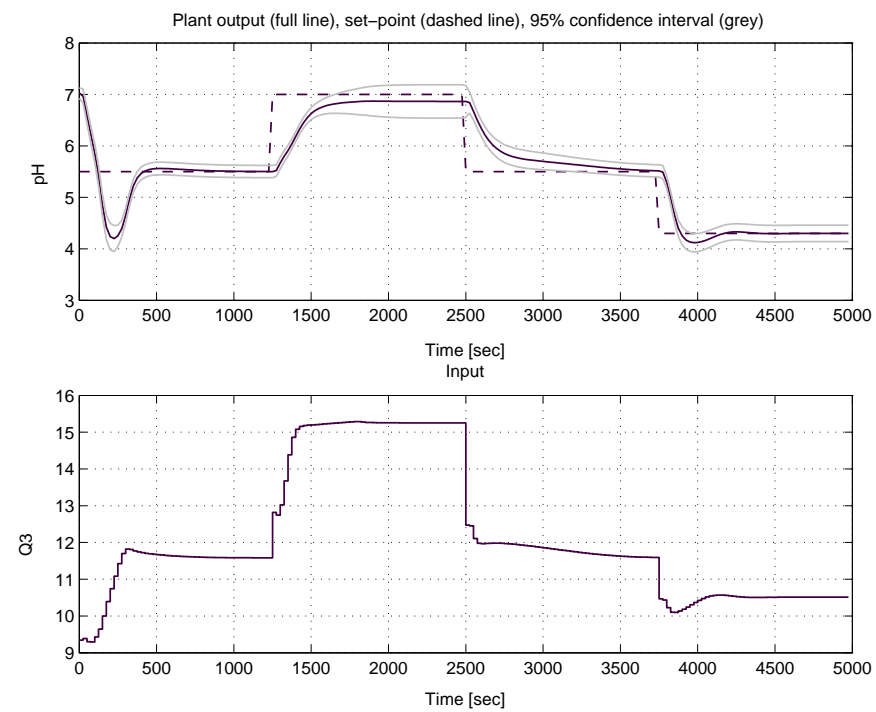

Fig. 7. Constrained case $\left(\sigma_{\max }=0.15\right)$ : response of GP model based control (upper figure) and control signal (bottom figure) 


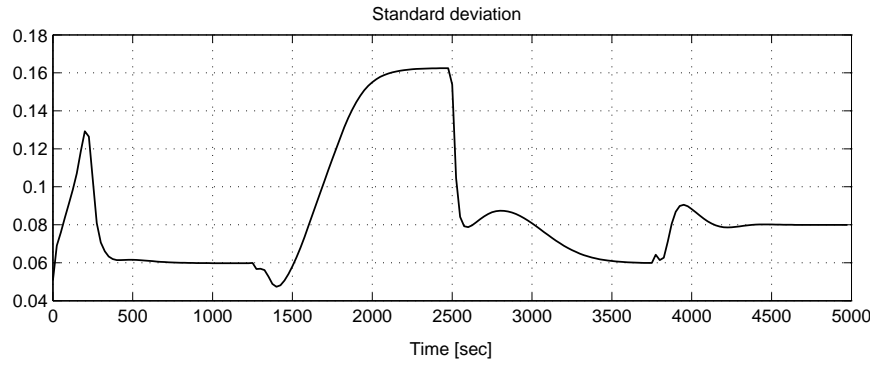

Fig. 8. Constrained case $\left(\sigma_{\max }=0.15\right)$ : standard deviation corresponding to the previous figure

complimented also with other constraints when necessary. The use of Gaussian process models makes it possible to include information about the trust in the model depending on the region. Incorporating this information enables a design of robust controller that will optimize action according to the validity of model. The paper indicates that using Gaussian process models offers an attractive possibility for control design that results in a controller with a higher level of robustness due to information contained in the model. It is necessary to stress that the presented control strategy represents only a feasibility test for Gaussian process application for model predictive control and additional efforts are necessary before this approach will be applicable in engineering practice. The principle shown in the paper is quite general and several modifications that accelerate computation can be used and are planned to be derived in the future.

\section{ACKNOWLEDGMENTS}

This work was made possible by EC funded MultiAgent Control Research Training Network HPRN-CT-199900107, and RM-S by EPSRC project GR/M76379/01, and Science Foundation Ireland grant 00/PI.1/C067. C. E. Rasmussen was supported by the German Research Council (DFG) through grant RA 1030/1. J. Kocijan acknowledges the support of Slovene Ministry of education, science and sport. The authors are grateful for comments and useful suggestions from Daniel Sbarbaro and Gregor Gregorčič.

\section{REFERENCES}

[1] Allgöwer F., Badgwell T.A., Qin S.J., Rawlings J.B., Wright S.J., Nonlinear predictive control and moving horizon estimation - an introductory overview, In: Frank, P.M (Edt.), Advances in control: highlights of ECC'99, Springer, 391-449, 1999.

[2] Allgöwer F., Zheng A. (Eds.), Nonlinear Model Predictive Control, Progress in system and control theory, Vol. 26, Birkhäuser Verlag, Basel, 2000.
[3] Girard A., Rasmussen C.E., Quinonero Candela, J. and Murray-Smith R., Gaussian Process Priors With Uncertain Inputs \& Application to Multiple-Step Ahead Time Series Forecasting, NIPS 15, Vancouver, Canada, MIT Press, 2003.

[4] Gregorčič G., Lightbody G., Internal model control based on a Gaussian process prior model, Proceedings of ACC'2003, Denver, CO, 4981-4986, 2003.

[5] Johansen T.A., Foss B.A., Sorensen A.V., Non-linear predictive control using local models - applied to a batch fermentation process, Control Eng. Practice, 3(3), 389-396, 1995.

[6] Henson M.A., Seborg D.E., Adaptive Nonlinear Control of a pH Neutralization Process, IEEE Trans. Control System Technology, Vol. 2, No. 3, 169-183, 1994

[7] Kavšek-Biasizzo K., Škrjanc I., Matko D., Fuzzy predictive control of highly nonlinear $\mathrm{pH}$ process, Computers \& chemical engineering, Vol. 21, Supp. 1997, S613-S618, 1997.

[8] Kerrigan E.C., Maciejowski J.M., Soft constraints and exact penalty functions in model predictive control, Control 2000 Conference, Cambridge, 2000.

[9] Kocijan J., Girard A., Banko B., Murray-Smith R., Dynamic Systems Identification with Gaussian Processes, Proceedings of 4th Mathmod, Vienna, 776-784, 2003.

[10] Kocijan J., Likar B., Banko B., Girard A., Murray-Smith R., Rasmussen C.E., A case based comparison of identification with neural network and Gaussian process models, Preprints of IFAC ICONS Conference, Faro, 137-142, 2003.

[11] Kouvaritakis B., Cannon M. (Eds.), Nonlinear predictive control, Theory and practice, IEE Control Engineering Series 61, IEE, 2001.

[12] Maciejowski J.M., Predictive control with constraints, Pearson Education Limited, Harlow, 2002.

[13] Murray-Smith, R., Girard, A., Gaussian Process priors with ARMA noise models, Irish Signals and Systems Conference, Maynooth, 147152, 2001.

[14] Murray-Smith, R and T. A. Johansen and R. Shorten, On transient dynamics, off-equilibrium behaviour and identification in blended multiple model structures, European Control Conference, Karlsruhe, BA-14, 1999.

[15] Murray-Smith R. and Sbarbaro D., Nonlinear adaptive control using nonparametric Gaussian process prior models, In: Proc. IFAC Congress, Barcelona, 2002.

[16] Nørgaard M., Ravn O., Poulsen N.K., Hansen L.K., Neural networks for modelling and control of dynamic systems, Springer, London, 2000.

[17] Qin S.J., Badgwell T.A., An overview of industrial model predictive control technology, In: Kantor J.C., Garcia C.E., Carnahan B. (Eds.) Fifthe international conference on Chemical process control, AChE and CACHE, 232-56, 1997.

[18] Qin S.J., Badgwell T.A., An overview of nonlinear model predictive control applications, In: Allgöwer F., Zheng A. (Eds.), Nonlinear model predictive control, Birkhauser Verlag, 369-392, 2000.

[19] Rasmussen C.E., Evaluation of Gaussian Processes and other Methods for Non-Linear Regression, Ph.D. Disertation, Graduate department of Computer Science, University of Toronto, Toronto, 1996.

[20] Solak, E., Murray-Smith R., Leithead, W.E., Leith, D.J., and Rasmussen, C.E., Derivative observations in Gaussian Process models of dynamic systems, NIPS 15, Vancouver, Canada, MIT Press, 2003.

[21] Williams C.K.I., Prediction with Gaussian processes: From linear regression to linear prediction and beyond, In: Learning in Graphical Models (Edt.: Jordan, M.I.), Kluwer Academic, Dordrecht, 599-621, 1998.

[22] Young R.E.,Bartusiak R.D., Fontaine, R.W., Evolution of an industrial nonlinear model predictive controller, Preprints on Chemical Process Control - CPC VI, CACHE, Tucson, AZ, 399-401, 2001

[23] Zheng A., Morari M., Stability of model predictive control with mixed constraints, IEEE Trans. Autom. Control, 40(19), 1818-1823, 1995. 